\title{
Sleep Disorders in Egyptian MS Patients: Clinical and Polysomnography Study Seham E. Abd Elsadek ${ }^{1}$, Manal H. Maabady ${ }^{1}$, Mohamed A. Shafik ${ }^{2 *}$ \\ ${ }^{1}$ Department of Neurology, Faculty of Medicine, Al-Azhar University, ${ }^{2}$ Department of Neurology, Faculty of Medicine, Ain Shams University, Egypt \\ *Corresponding author: Mohamed A. Shafik, Mobile: 01003088969, Email: mshafik@ gmail.com
}

\begin{abstract}
Background: multiple sclerosis (MS) is an inflammatory disorder of the brain, spinal cord, and optic nerves. MS affects more than two million people worldwide; sleep disorders are very common in the general population; their high prevalence rate suggests that MS patients will certainly be suffering from comorbid sleep disorders.
\end{abstract}

Objective: study the prevalence of sleep disorders among MS patients by clinical and Polysomnography (PSG) studies.

Patients and Methods: twenty-five MS patients and 12 healthy controls were examined by expanded disability status scale (EDSS) for functional disability, Pittsburg sleep quality index (PSQI) for sleep quality, Epworth sleepiness scale (ESS) for excessive daytime sleepiness, international restless leg syndrome scale (IRLSS) for restless led syndrome (RLS). PSG studies were done for all subjects for assessment of quality of sleep and sleep parameters. Magnetic Resonance Imaging (MRI) Brain was done for MS patients g to subtype it according to McDonald criteria 2010.

Results: seventy six percent of MS patients had poor sleep quality according to PSQI (8.6 \pm 3.7$)$. While 44\% had excessive daytime sleepiness according to ESS (12.2 \pm 3.6$)$ and 40\% had RLS according to IRLSS. Patients with progressive MS (PMS) either primary (PPMS) or secondary (SPMS) subtypes had poorer sleep quality index and excessive daytime sleepiness compared to relapsing remitting MS (RRMS) subtype. PSG study showed decreased total sleep time (TST) and sleep efficiency index in MS patients with prolonged sleep latency and decreased rapid eye movement (REM) latency and higher total arousal index and periodic limb movement (PLM) index than control. EDSS of MS patients had positive correlation with PSQI and ESS. Also EDSS had inverse correlation with TST and sleep efficiency index on PSG.

Conclusion: sleep disorders are prevalent among MS patients. PPMS and SPMS patients showed more prevalence of sleep disorder than RRMS with poorer sleep parameters on PSG.

Keywords: Multiple Sclerosis (MS), Polysomnogram (PSG), Pittsburg sleep quality index (PSQI), Epworth sleepiness scale (ESS), international restless leg syndrome scale (IRLSS), expanded disability status scale (EDSS).

\section{INTRODUCTION}

Complex interactions between genetic and environmental factors are implicated in the pathogenesis of Multiple sclerosis (MS) which is an inflammatory disorder of the brain, spinal cord, and optic nerves of unknown etiology ${ }^{(\mathbf{1}, 2,3)}$.MS develops between the ages of 20 and 40years with average age of 30years and women are twice more affected than men ${ }^{(4)}$.Two million people and more have MS worldwide with the highest prevalence rate in North America and Europe ${ }^{(4)}$.

MS considered to be the most common disabling non-traumatic neurological conditions in young adults according to the World Health Organization (WHO) ${ }^{(5,6)}$. Sleep disorders are very common in the general population. The prevalence of sleep apnea syndrome is $4 \%$ among men and $2 \%$ among women in the workforce ${ }^{(7)}$. While, the prevalence of restless leg syndrome (RLS) is $10.6 \%$ with women more affected than men ${ }^{(8)}$. Insomnia is prevalent among 6 to $18 \%$ of the general population ${ }^{(9)}$. The high prevalence rate of sleep disorders suggests that MS patients will certainly be suffering from comorbid sleep disorders as does a large part of the general population. Also; A causative link between MS and some sleep disorders have been postulated; for example, the prevalence rate of RLS is four times higher in MS than in the general population (10). And some MS patients present narcolepsy-like symptoms. But; it is difficult to differentiate if these sleep disorders are due to MS or independent of it (e.g. idiopathic narcolepsy) ${ }^{(\mathbf{1 1})}$. sleep disorders were present among 74\% of consecutive MS patients in a recent polysomnographic cross sectional study (49 out of 66 patients) ${ }^{(\mathbf{1 2})}$.

\section{AIM OF THE STUDY}

- To assess the quality of sleep and prevalence of sleep disorders in patients with MS by clinical and PSG studies.

- To evaluate the severity of sleep disorders between RRMS and PMS patients.

\section{PATIENTS AND METHODS}

Study Design: A case control study.

Study Period: Between October 2015 and January 2017. 
Study Place: Al Zahraa (Al-Azhar) University hospital.

Study Cohort: The patients (Cases) group included 25 patients diagnosed with multiple sclerosis (MS) according to McDonald 2010 criteria ${ }^{(13)}$ selected from inpatient ward and outpatient clinic of neurology department of $\mathrm{Al}$ Zahraa University hospital and the control group included 12 apparently healthy volunteers of matched age and sex.

\section{Inclusion criteria:}

Patients diagnosed as definite MS according to revised McDonald criteria 2010 eitherRRMS or PMS subtypes (PPMS or SPMS).

\section{Exclusion criteria:}

Patients with premorbid systemic diseases (renal, hepatic, Cardiac, Pulmonary and DM), Other neurological diseases (Stroke, Epilepsy, Dementia and Neuromuscular diseases), Iron deficiency Anemia, Other chronic diseases like malignances, patients with primary sleep disorders prior to diagnosis of MS, patients taking medications which interfere with normal sleep habit and any disease known to cause sleep disorders were excluded from this study.

\section{Methods:} subjected to:

All patients included in this study were

- Full medical history with general examination.

- Neurological assessment including history and complete neurological examination focusing on age at onset, duration of illness, course of disease and annual relapse rate.

- Neurological disability was evaluated and scored by using the Expanded Disability Status Scale (EDSS) ${ }^{(14)}$.

- Laboratory investigations were done for patients and controls including: Complete blood picture (CBC), Fasting blood glucose levels (FBS), Lipid profile, Liver function (LFTs) and Kidney function tests (KFTs) to exclude systemic diseases.

- Magnetic resonance imaging (MRI) of the brain and spine were performed for patients using General Electric Medical System Signal 1.5 Tesla, the results of T1, T2 -weighted spin echo images, FLAIR pulse sequences and post contrast $\mathrm{T} 1$ sequences were obtained and all patients fulfill the MRI criteria for definite MS according to Mc Donald 2010 criteria $^{(13)}$.

- Sleep is assessed for all patients and control groups by the:

- Pittsburgh Sleep Quality Index (PSQI) ${ }^{(15)}$ to assess quality of sleep, a score of (5) or more is indicative of poor sleep quality.

- Epworth Sleepiness Scale (ESS) ${ }^{(15)}$ to assess excessive daytime sleepiness, a score of (10) or more is indicative of hyper somnolence.
International Restless Legs Syndrome Rating Scale (IRLSS) ${ }^{(16)}$ for the presence of restless legs syndrome (RLS).

All patients and controls were subjected to nocturnal Polysomnography (PSG) examination (from $10 \mathrm{pm}$ to 7am), PSG is a recording of multiple parameters relevant to sleep. The PSG was performed in our sleep laboratory using (40 channel Neuronspectrum-5equipment, Russia), in a sound-attenuated room with temperature control. Patients were required to maintain their daily routine habits, including diet and therapeutic drug intake. PSG involves monitoring and recording Electroencephalogram (EEG), Electrooculogram (EOG), electromyography (EMG) and other physiologic data used to analyze sleep architecture, cardiopulmonary function and limb movements in sleep.

Analysis of sleep data: PSG was scored according to slandered manual for staging normal sleep of Rechtschataffen and Kales ${ }^{(17)}$. The analysis of sleep data consist of report generation and hypnogram.

The recording was scored:

- Sleep stages, they were scored according to the standard scoring system for sleep stages done by Rechtschataffen and Kales ${ }^{(17)}$.

- Time in bed (TIB).

- Total sleep time (TST).

- Sleep efficiency (TST / Time in bed X 100).

- Sleep onset latency.

- REM latency.

- Percentage (\%) of TST in each stage $\left(\mathrm{S}_{1}, \mathrm{~S}_{2}\right.$, SWS and REM).

- Number of awakenings more than 2 minute duration.

- Attacks of central apnea.

- Attacks of obstructive apnea.

- Attacks of hypopneas.

- Apnea/Hypopnea index (AHI); equals number of episodes of apnea and hypopnea per hour of sleep. AHI more than five per hour (5/hour) is considered pathologic.

- Periodic Limb Movement index (PLM): PLM index equals the number of PLM episodes during the night/ TST. A PLM index above fifteen per hour (15/hour) is considered pathologic.

- Lowest Oxygen saturation.

- Oxygen desaturation events/hour: No. of oxygen desaturation events / TST x 100 . Where, oxygen desaturation event is detected when the oxygen saturation fell at least $4 \%$.

\section{Statistical analysis}

Data were analyzed using Statistical Program for Social Science (SPSS) version 18. Quantitative 
data were expressed as mean \pm standard deviation (SD). Qualitative data were expressed as frequency and percentage. Independent-samples t-test was used when comparing between two means, and one way test (ANOVA) was used when comparing between more than two groups.

Chi-square $\left(\chi^{2}\right)$ test was used in order to compare proportions between two qualitative parameters, Pearson's correlation coefficient (r) test was used to describe the degree of correlation between two variable, the sign of correlation coefficient $(+,-)$ defines the direction of the relationship either positive or negative. The probability and significance ( $\mathrm{p}$ value $<0.05$ was considered significant, $\mathrm{p}<0.001$ was considered high significant and $\mathrm{p}>0.05$ was considered non-significant).

\section{Ethical Consideration:}

All subjects were informed of the general aim of the study and their participation was fully voluntary. Informed consent had been obtained and approved by the ethics committee for clinical research of faculty of medicine Azhar University for girls.
The study was carried out on 25patients with definite MS. Eleven males (44\%) and 14 female $(56 \%)$ with mean age $(32.7 \pm 9.7)$ years. The mean age at the onset of disease was (25.6 \pm 8.7$)$ years and duration of disease ranged from 2 years to 12 years with mean $(6.4 \pm 2.4)$ years with annual relapse rate (ARR) ranged from one attack per year to 3 attacks per year with mean $(1.7 \pm 0.8)$ attacks per year. The mean score of Expanded Disability Status Scale (EDSS) was (3.6 \pm 0.85 ). The patients group divided into three MS subtypes according to McDonald criteria with 14 RRMS patients (56\%); 7 SPMS patients (28\%) and 4 PPMS Patients (16\%).

The site of MS Lesions in MRI Brain were variable; where $22(88 \%)$ of the patients have periventricular lesions; While $12(48 \%)$ have Brain stem lesions; 8 patients $(32 \%)$ have cerebellar lesions; 10 patients $(40 \%)$ had contrast enhancement lesions; 6 patients $(24 \%)$ had black holes in T1w images and Spine lesions were present in 6 patients (34\%).

As regards RLS; 10 patients $(40 \%)$ had restless leg syndrome (RLS) according to the IRLSS,Table(1)

\section{RESULTS}

Table (1): Demographic and Descriptive data of the patients group

\begin{tabular}{||c|c|}
\hline \multicolumn{1}{|c|}{ Demographic Data } & Mean \pm SD \\
\hline Age (years) & $32.7 \pm 9.7$ \\
\hline Sex (Male/ Female) & $11(44 \%) / 14(56 \%)$ \\
\hline Clinical data of the patients & \\
\hline Type of MS & $14(56 \%)$ \\
\hline RRMS & $7(28 \%)$ \\
\hline SPMS & $4(16 \%)$ \\
\hline PPMS & $25.6 \pm 8.7$ \\
\hline Age at onset & $6.4 \pm 2.4$ \\
\hline Duration of MS /years & $1.7 \pm 0.8$ \\
\hline Annual relapse rate & $3.6 \pm 0.85$ \\
\hline EDSS & $22(88 \%)$ \\
\hline Distribution of MRI brain lesions (\%) & $12(48 \%)$ \\
\hline Periventricular & $8(32 \%)$ \\
\hline Brain stem & $10(40 \%)$ \\
\hline Cerebellum & $6(24 \%)$ \\
\hline Enhancement lesions & $6(24 \%)$ \\
\hline Black holes & \\
\hline MRI Spine lesions & $10(40 \%)$ \\
\hline Restless leg syndrome (IRLS)scale & $15(60 \%)$ \\
\hline Patients with RLS & \\
\hline Patients without RLS & \\
\hline
\end{tabular}

MS; Multiple sclerosis, RRMS; Relapsing Remitting MS, SPMS; Secondary Progressive MS, PPMS; Primary Progressive MS, EDSS; Expanded disability status scale. RLS; Restless leg syndrome

Nineteen MS patients (76\%) had poor sleep quality according to the Pittsburg sleep quality index (PSQI) with mean score $(8.6 \pm 3.7)$ and $11 \mathrm{MS}$ patients $(44 \%)$ had excessive daytime sleepiness (i.e. Hypersomnia) according to the Epworth sleepiness scale (ESS) with mean score (12.2 \pm 3.6$)$ with highly statistically significant difference between Patients and control group ( $\mathrm{P}<0.001 ; 0.009$ respectively), Table (2). 
Table (2): Comparison between patients and control as regard quality of sleep and excessive daytime sleepiness

\begin{tabular}{||l|c|c|c|c||}
\hline \hline & $\begin{array}{c}\text { Patients } \\
(\mathbf{n = 2 S})\end{array}$ & $\begin{array}{c}\text { Control } \\
(\mathbf{n = 1 2})\end{array}$ & t test & p-value \\
\hline \hline Pittsburgh Sleep Quality & $\mathrm{n} 19(76 \%)$ & $4.5 \pm 1.9$ & $\mathbf{3 . 5 4 1}$ & $\mathbf{0 . 0 0 1}$ \\
$\begin{array}{l}\text { Index } \\
\text { Mean } \pm \text { SD }\end{array}$ & $\begin{array}{c}\mathrm{n} 11(44 \%) \\
12.2 \pm 3.6\end{array}$ & $8.83 \pm 2.8$ & $\mathbf{2 . 7 8 2}$ & $\mathbf{0 . 0 0 9}$ \\
\hline $\begin{array}{l}\text { Epworth Sleepiness Scale } \\
\text { Mean } \pm \text { SD }\end{array}$ & & \\
\hline
\end{tabular}

Patients with progressive MS (PMS) either primary or secondary subtypes had poorer sleep quality index and excessive daytime sleepiness compared to relapsing remitting MS (RRMS) subtype with highly statistically significant difference $(\mathrm{P}<0.001)$. Seventy five percent of PPMS patients had RLS compared to $42 \%$ of SPMS and $35 \%$ of RRMS patients which is statistically significant $(\mathrm{P}<0.05)$, Table (3).

Table (3): Comparison between RRMS, SPMS and PPMS patients as regard quality of sleep, excessive daytime sleepiness and Restless Legs Syndrome

\begin{tabular}{|c|c|c|c|c|c|}
\hline & $\begin{array}{l}\text { RRMS } \\
(\mathrm{n}=14)\end{array}$ & SPMS $(n=7)$ & $\operatorname{PPMS}(n=4)$ & & p-value \\
\hline $\begin{array}{l}\text { Pittsburgh Sleep Quality } \\
\text { Index (PSQI) } \\
\text { Mean } \pm \text { SD }\end{array}$ & $6.3 \pm 2.1$ & $10.2 \pm 2.8$ & $14.2 \pm 1.7$ & $F=20.2$ & 0.001 \\
\hline $\begin{array}{l}\text { Epworth Sleepiness Scale } \\
\text { (ESS) Mean } \pm \text { SD }\end{array}$ & $10.2 \pm 2.6$ & $13.5 \pm 2.4$ & $17.5 \pm 1.7$ & $F=16.0$ & 0.001 \\
\hline $\begin{array}{l}\text { International Restless Legs } \\
\text { Syndrome Rating Scale. N } \\
(\%) \\
\text { With RLS Without RLS }\end{array}$ & $\begin{array}{l}5(35.8 \%) \\
9(64.2 \%)\end{array}$ & $\begin{array}{l}3(42.8 \%) \\
4(57.2 \%)\end{array}$ & $\begin{array}{l}3(75 \%) \\
1(25 \%)\end{array}$ & X2 5.368 & 0.02 \\
\hline
\end{tabular}

PSG shows that the total sleep time and sleep efficiency index are highly statistically significant decreased in MS patient in comparison to control group $(\mathrm{P}<0.001)$.

Additionally, patients with MS had prolonged sleep latency and decreased REM latency with higher total arousal index and periodic limb movement (PLM) index than control group with highly statistically significant differences $(\mathrm{P}<0.001 ; 0.031 ; 0.006$ and 0.021 respectively).

Regarding sleep stages; the MS patients had prolonged REM sleep stage duration in comparison to control with highly statistically significant difference $(\mathrm{P}<0.001)$. While in NonREM sleep stages; MS patients had prolonged stage 1 and 2 and shortened slow wave sleep (SWS) stage duration compared to controls which is statistically significant $(\mathrm{P}<0.03 ; 0.027$ respectively).

According to sleep respiratory events and oxygen saturation, MS patients showed more obstructive and central apnea events than controls. But; central apneas showed statistically significant increase $(\mathrm{p}<0.035)$.Also; MS patients showed statistically significant lower $02 \quad(\%)$ and statistically highly significant higher oxygen desaturation index $(\%)$ than controls $(\mathrm{P}<0.004$; 0.000 respectively), Table 
Table (4): Comparison between patients and control groups as regard PSG sleep parameters

\begin{tabular}{|l|c|c|c|c||}
\hline Sleep Parameters & $\begin{array}{c}\text { Patients group } \\
(\mathbf{n = 2 S}) \\
(\text { Mean } \pm \text { SD) }\end{array}$ & $\begin{array}{c}\text { Controls } \\
\text { group(n=12) } \\
\text { (Mean } \pm \text { SD) }\end{array}$ & t-test & p-value \\
\hline \hline Time in bed (TIB) (hrs.) & $6.8 . \pm 0.88$ & $6.7 \pm 1.03$ & 0.025 & 0.980 \\
\hline Total sleep time(TST)(hrs) & $4.50 \pm 1.09$ & $5.85 \pm .934$ & 3.650 & $\mathbf{0 . 0 0 1}$ \\
\hline Sleep efficiency index \% & $65.40 \pm 14.03$ & $85.66 \pm 9.04$ & 4.548 & $\mathbf{0 . 0 0 0}$ \\
\hline Number of arousal >2min & $5.44 \pm 3.1$ & $2 \pm 1.044$ & 3.424 & $\mathbf{0 . 0 0 6}$ \\
\hline Sleep latency & $31.56 \pm 12.89$ & $17.83 \pm 6.07$ & 3.488 & $\mathbf{0 . 0 0 1}$ \\
\hline $\begin{array}{l}\text { Rapid Eye Movement(REM) } \\
\text { latency }\end{array}$ & $69.84 \pm 24.5$ & $87.1 \pm 14.80$ & 2.246 & $\mathbf{0 . 0 3 1}$ \\
\hline Non REM Sleep Stages \% & & & & \\
\hline Stage 1 & $10.8 \pm 8.0$ & $5.8 \pm 1.40$ & 2.148 & $\mathbf{0 . 0 3}$ \\
\hline Stage 2 & $38.16 \pm 12.26$ & $32.46 \pm 11.00$ & 1.376 & 0.177 \\
\hline Slow wave sleep & $17.32 \pm 5.85$ & $22.25 \pm 4.84$ & 2.514 & $\mathbf{0 . 0 2 7}$ \\
\hline REM sleep stage \% & $22.66 \pm 3.84$ & $16.24 \pm 5.72$ & 3.514 & $\mathbf{0 . 0 0 1}$ \\
\hline Respiratory events & & & & \\
\hline Central apnea & $15.32 \pm 16.36$ & $4.41 \pm 6.51$ & 2.211 & $\mathbf{0 . 0 3 4}$ \\
\hline Obstructive apnea & $3.88 \pm 5.11$ & $2.81 \pm 3.21$ & 1.250 & $\mathbf{0 . 0 9 1}$ \\
\hline Mixed apnea & $2.20 \pm 3.23$ & $1.36 \pm 2.06$ & 1.163 & $\mathbf{0 . 1 8 3}$ \\
\hline Apnea hypopnea indexlhour & $6.88 \pm 7.622$ & $5.45 \pm 2.33$ & 1.297 & $\mathbf{0 . 1 7 6}$ \\
\hline Oxygen saturation & & & & \\
\hline Baseline O2 (\%) & $93.8 \pm 3.6$ & $95.0 \pm 2.4$ & 0.892 & 0.353 \\
\hline Lowest O2 (\%) & $72.7 \pm 15.1$ & $88.6 \pm 6.7$ & 4.038 & $\mathbf{0 . 0 0 4}$ \\
\hline Oxygen desaturation index (\%) & $35.88 \pm 19.4$ & $9.00 \pm 6.36$ & 4.646 & $\mathbf{0 . 0 0 0}$ \\
\hline Periodic leg movement(PLM) & & & & \\
\hline disorder & & & & $\mathbf{0 . 0 0 4}$ \\
\hline Number of periodic leg movements & $32.1 \pm 30.94$ & $9.50 \pm 13.37$ & 3.106 & $\mathbf{0 . 0 2 1}$ \\
\hline periodic leg movements index & $9.24 \pm 8.74$ & $4.166 \pm 6.30$ & 2.417 & \\
\hline PLM lhour & & & & \\
\hline
\end{tabular}

Progressive PMS patients either PPMS or SPMS had decreased total sleep time (TST) and sleep efficiency index in comparison to RRMS with highly statistically significant difference $(\mathrm{P}<0.000)$. While; SPMS patients and RRMS showed higher total arousal index than PPMS patients with statistically significant difference $(\mathrm{p}<0.034)$. Yet; No statistically significant difference as regard other sleep parameters, Table (5).

Table (5): Comparison between RRMS, SPMS and PPMS patients as regard PSG sleep parameters

\begin{tabular}{||l|l|l|l|l|l||}
\hline \multicolumn{1}{|c|}{ PSG Sleep Parameters } & $\begin{array}{c}\text { RRMS (n=14) } \\
(\text { Mean } \pm \text { SD) }\end{array}$ & $\begin{array}{l}\text { SPMS (n=7) } \\
(\text { Mean } \pm \text { SD) }\end{array}$ & $\begin{array}{c}\text { PPMS( }=4) \\
(\text { Mean } \pm \text { SD) }\end{array}$ & \multicolumn{1}{|c||}{ t-test } & \multicolumn{1}{|c|}{ p-value } \\
\hline \hline Total sleep time(TST)(hrs) & $5.22 \pm 0.723$ & $3.42 \pm .0 .672$ & $3.87 \pm 0.853$ & 15.995 & $\mathbf{0 . 0 0 0 * *}$ \\
\hline Sleep efficiency index \% & $73.87 \pm 8.33$ & $56.70 \pm 15.13$ & $51.00 \pm 5.35$ & 11.004 & $\mathbf{0 . 0 0 0 * *}$ \\
\hline Number of arousal >2min & $3.85 \pm 2.24$ & $6.85 \pm 4.09$ & $2.25 \pm 2.06$ & 3.977 & $\mathbf{0 . 0 3 4}$ \\
\hline Sleep latency & $26.35 \pm 11.16$ & $37.85 \pm 12.54$ & $38.75 \pm .13 .69$ & 3.037 & $\mathbf{0 . 0 6 8}$ \\
\hline REM latency & $76.50 \pm 23.54$ & $58.42 \pm 28.16$ & $66.50 \pm 17.55$ & 1.345 & $\mathbf{0 . 2 8 1}$ \\
\hline $\begin{array}{l}\text { Number of periodic leg } \\
\text { movements }\end{array}$ & $25.57 \pm 31.62$ & $42.14 \pm 31.60$ & $37.75 \pm 29.15$ & 0.327 & 0.493 \\
\hline $\begin{array}{l}\text { periodic leg movements index } \\
\text { hour }\end{array}$ & $6.92 \pm 8.42$ & $11.42 \pm 8.904$ & $13.50 \pm 9.255$ & 1.202 & 0.320 \\
\hline
\end{tabular}

Functional disability among MS patients assessed by expanded disability status score (EDSS) had positive correlation with Pittsburgh Sleep Quality (PSQ) Index score and excessive daytime sleepiness assessed by Epworth sleepiness scale (ESS) with statistically significant difference ( $<<0.007 ; 0.008$ respectively). Also EDSS had negative (inverse) correlation with total sleep time (TST) and sleep efficiency index on PSG ( $p<0.002 ; 0.032$ respectively), Table (6) and Figures (1,2 and 3). 
Table (6): Correlation between EDSS and Pittsburg sleep quality index, Epworth sleepiness scale, Total sleep time and Sleep efficiency index.

\begin{tabular}{|c|c|c|}
\hline \multirow{2}{*}{ Parameters } & \multicolumn{2}{|c|}{ EDSS } \\
\cline { 2 - 3 } & r & p-value \\
\hline \hline Pittsburg sleep quality index & 0.529 & $\mathbf{0 . 0 0 7}$ \\
\hline Epworth sleepiness scale & 0.529 & $\mathbf{0 . 0 0 8}$ \\
\hline Total sleep time & -0.622 & $\mathbf{0 . 0 0 2}$ \\
\hline Sleep efficiency index & -0.453 & $\mathbf{0 . 0 3 2}$ \\
\hline
\end{tabular}

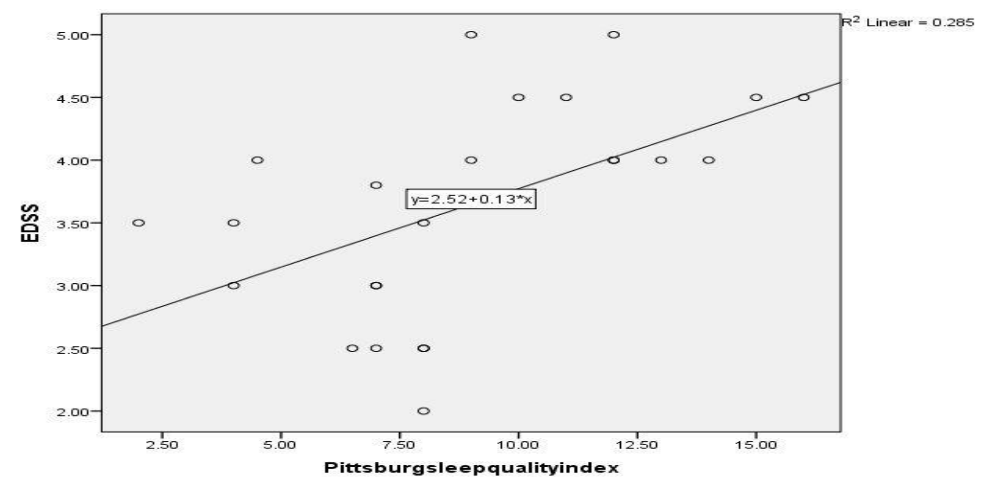

Figure (1): Correlation between EDSS and Pittsburgh Sleep Quality Index

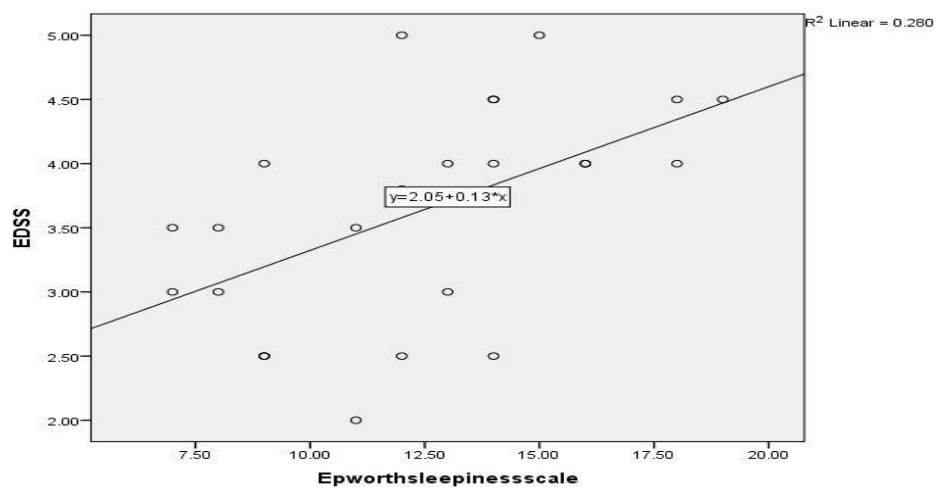

Fig (2) Correlation between EDSS and Epworth Sleepiness Scale

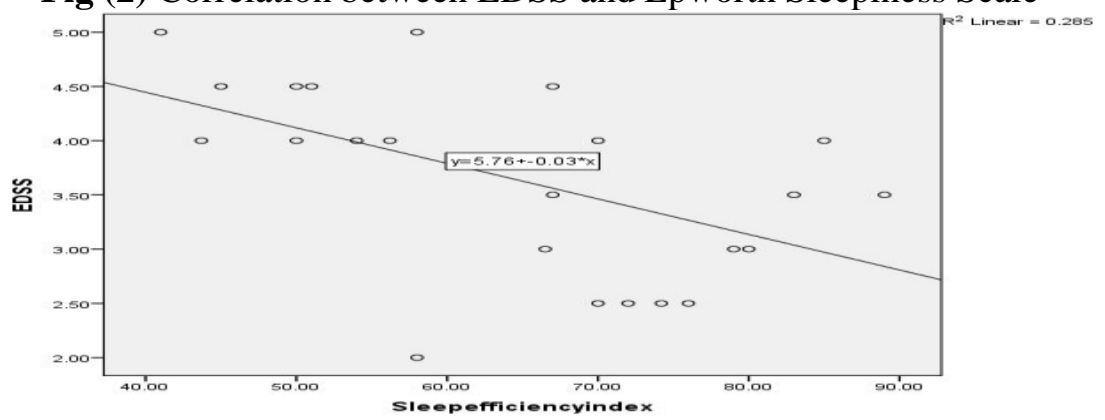

Figure (3): Correlation between EDSS and Sleep efficiency index 


\section{DISCUSSION}

Insomnia in the ICD-10 of the World Health Organization (WHO) is defined as incapability to initiate sleep, or as disrupted sleep or early morning awakenings); including organic i.e., secondary to MS and non-organic insomnia as well ${ }^{(\mathbf{1 8})}$.

The ICSD-3 defines insomnia as a persistent difficulty with sleep initiation, duration, consolidation, or quality that occurs despite adequate opportunity and circumstances for sleep, and results in some form of day impairment ${ }^{(\mathbf{1 9})}$.

The prevalence of insomnia in MS seems to be higher than in the general population, but studies comparing the prevalence of insomnia in MS with the general population in systematic manner are lacking today. Approximately one fourth to one half of MS patients are suffering from insomnia. Albeit; we do not have robust data confirming the hypothesis that the prevalence of insomnia is higher in MS than in the general population ${ }^{(20)}$.

Another study according to ICSD-2 criteria reporting insomnia in $25 \%$ of MS patients ${ }^{(12)}$.

Brass and colleagues found moderate to severe insomnia in $31.6 \%$ of 2375 MS patients using validated questionnaire ${ }^{(\mathbf{2 1})}$.

Leona vicius and colleagues found sleep disturbances in $45.3 \%$ of MS patients using the Medical Outcomes Study Sleep (MOSS) scale ${ }^{(22)}$.

Tachibana and colleagues reported sleep problems in 53.6 \% of MS patients including discomfort in the legs, snoring, nocturia, and sleep apnea ${ }^{(23)}$.

The current study revealed poor sleep Quality among $76 \%$ of MS patients according to the Pittsburg sleep quality index (PSQI) with mean score $(8.6 \pm 3.7)$ (Table 2).

Polysomnographic parameters supported this finding showing that total sleep time and sleep efficiency index are highly statistically significant decreased in MS patients in comparison to control group; (P<0.001and 0.000) (Table 4).

Additionally, patients with MS had prolonged sleep latency and decreased REM latency with higher total arousal index and periodic limb movement index than control group $(P<0.001 ; 0.031 ; 0.006$ and 0.021 respectively)(Table 4 ).

Regarding Sleep stages; MS patients had prolonged REM stage and stage 1 of non REM sleep with decreased duration of slow wave sleep (SWS) stage compared to control $(p<0.001 ; 0.05$ and 0.027 respectively) (Table 4).

Regarding MS subtypes; patients with progressive MS (PMS) either primary PPMS or secondary SPMS subtypes had poorer sleep quality index by PSQI compared to relapsing remitting (RRMS) subtype which is highly statistically significant $(P<0.001)$ (Table 3).
Polysomnographic (PSG) parameters supported these results showing that progressive MS patients had decreased total sleep time and sleep efficiency index with higher total arousal index than RRMS patients with highly statistically significant difference $(P<0.001 ; 0.001$ and 0.023 respectively). Yet; no statistically significant difference as regard sleep and REM latency (Table 5)

Obstructive sleep apnea (OSA) known also as obstructive sleep apnea hypopnea (OSAH) syndrome is the most common sleep -related breathing disorder (SRBD). Other less frequent SRBDs are central sleep apnea syndrome (CSA), central alveolar hypoventilation ${ }^{(19)}$, obesity hypoventilation syndrome (OHS), complex sleep apnea with persisting central apneas after starting continuous positive airway pressure (CPAP) therapy and upper airway resistance syndrome (UARS), has been described as a new SRBD requiring esophageal manometry to detect an increase of inspiratory esophageal pressure ${ }^{(24,25)}$. To date, we do not have exact data about the prevalence of OSA or other SRBDs in MS patients ${ }^{(26)}$.

Kaminska and colleagues found OSA in 58 $\%$ of MS patients and $49 \%$ of healthy controls using the American Academy of Sleep Medicine (AASM) research criteria (apnea or hypopnea for 10s associaed with arousal or desaturation $4 \%$ ) and an apnea/ hypopnea index (AHI) cutoff of $15 / \mathrm{h}$. when using only desaturation associated hypopnea/apnea (without arousal associated hypopnea/apnea), $11 \%$ were suffering from OSA ${ }^{(27,28)}$.

Veauthier and colleagues found SRBD in 12 $\%$ of consecutive MS patients using the older AASM Task force criteria from 1999 and an AHI cutoff of 10/h ${ }^{(12,29)}$.

Kallweit and colleagues Investigated 69 fatigued MS patients with over-night respirography, SRBD was present in $41 \%{ }^{(30)}$.

Braley and colleagues Investigated $30 \mathrm{MS}$ patients and 30 healthy controls (HC) by polysomnography using a lower AHI-cut-off of 5/. OSA was among $80 \%$ of MS patients and $63 \%$ of controls ${ }^{(31)}$.

Chen and colleagues Investigated 21 MS patients and 10 healthy controls using the same low AHI cutoff of $5 / \mathrm{h}$, and none of the patients or controls had an AHI 5/h ${ }^{(32)}$.Braley and colleagues retrospectively analyzed the polysomnographic data of 48 MS patients compared with 48 controls matched for age, gender, and body mass index (BMI). OSA as well as CSA were more severe in MS patients than in controls ${ }^{(33)}$.

In the current study PSG findings revealed a statistically significant difference between MS patients and control groups regarding sleep respiratory events and oxygen saturation particularly central apnea events which showed statistically significant increase with 
mean $(15.32 \pm 16.36)$ among MS patients $(\boldsymbol{p}<\mathbf{0 . 0 3 5})$ (Table 4).

Also; MS patients showed statistically significant lower $\mathrm{O} 2(\%)$ and statistically highly significant higher oxygen desaturation index $(\%)$ than controls $(P<0.004 ; 0.000$ respectively) (Table 4$)$.

Restless Leg Syndrome in The ICSD-3 is defined as "an urge to move the legs, usually accompanied by or thought to be caused by uncomfortable and unpleasant sensations in the legs" which (i) begin or worsen during periods of rest or inactivity, (ii) are relieved by movement, and (iii) occur (exclusively or predominantly) in the evening or night ${ }^{(19)}$.

Moreover, the ICSD-3 requires that these features are not accounted for as symptoms of another medical or behavioral condition and cause distress, sleep disturbances, or daytime impairment. RLS may be idiopathic or symptomatic of an underlying condition (i.e., chronic renal failure, anemia, and iron deficiency) ${ }^{(19)}$.

The prevalence of RLS is four times higher in MS than in the general population ${ }^{(\mathbf{1 0})}$.

Deficiency and subsequent dopaminergic dysfunction play a major role in the etiology of RLS ${ }^{(30)}$.PLMD is considered to be phenotype of RLS. Low iron stores and decreased ferritin serum levels were found in periodic limb movement disorder (PLMD) as well $(19,35$ )

The current study revealed that $40 \%$ of MS patients had RLS according to the International Restless Leg Syndrome Rating Scale (IRLS). (Table 1)

We found that the prevalence of RLS is more among PMS than RRMS. Where $75 \%$ of PPMS patients had RLS compared to $42 \%$ of SPMS and $35 \%$ of RRMS patients which is statistically significant $(\boldsymbol{P}<$ 0.05). (Table 3)

PSG results revealed that MS patients had higher PLM index than control group which is statistically significant $(\boldsymbol{P}<0.021)$ (Table 4).Yet; There was no statistically significant difference between different subtypes of MS regarding PLM index (Table 5).

Narcolepsy type 1 (hypocretin deficiency syndrome) is mainly characterized by excessive daytime sleepiness (EDS) and signs of REM sleep dissociation, the most specific of which is cataplexy, and it is caused by a deficiency of hypothalamic neuropeptide hypocretin (orexin) signaling ${ }^{(\mathbf{1 9})}$

Apart from EDS for at least 3 months, the diagnosis of narcolepsy type 1 requires either the presence of cataplexies, reduced sleep latencies in the multiple sleep latency test (MSLT) of $\leq 8 \mathrm{~min}$ in combination with two or more sleep-onset REM periods (SOREMP) or reduced hypocretin-1 (HCRT-1) levels in the cerebrospinal fluid (CSF) (either $\leq 110$ $\mathrm{pg} / \mathrm{mL}$ or $<1 / 3$ of mean values obtained in normal subjects with the same standardized assay) $(\mathbf{1 9}, \mathbf{3 6}$. Narcolepsy type 2 similarly requires the presence of EDS for at least 3 months but in contrast to narcolepsy type 1 the absence of cataplexies and the absence of reduced HCRT1. Additional criteria according to the ICSD-3 are reduced sleep latencies in the MSLT of $<8 \mathrm{~min}$ and two or more SOREMP ${ }^{(\mathbf{1 9})}$.

It is not known for certain if the prevalence of narcolepsy is higher in MS patients than in the general population.To date, robust epidemiological data and powerful studies investigating consecutive MS patients by MSLT in a systematic manner are lacking ${ }^{(20)}$.

Our study revealed that $11 \mathrm{MS}$ patients (44\%) had excessive daytime sleepiness (i.e. Hypersomnia) according to the Epworth sleepiness scale (ESS) with mean score $(12.2 \pm 3.6)$ with highly statistically significant difference $(\mathbf{P}<\mathbf{0 . 0 0 1})$ between Patients and control group (Table 2).

Patients with progressive MS (PMS) either primary or secondary subtype had excessive daytime sleepiness (EDS) compared to relapsing remitting (RRMS) subtype with highly statistically significant difference $(\boldsymbol{P}<0.001)$ (Table 3).

Our study revealed that MS patients had shorter REM latency $(69.84 \pm 24.5)$ in PSG than control group $(87.1 \pm 14.80)$ which is statistically significant $(\boldsymbol{P}<0.031)$ (Table 4).

Despite of PMS patients showed shorter REM latency either SPMS $(58.42 \pm 28.16)$ or PPMS

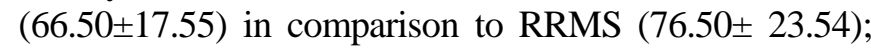
this was not statistically significant different; (Table 5).

Regarding Functional Disability among MS patients with Sleep disorders There is clear positive correlation between functional disability of MS patients assessed by EDSS and Quality of sleep assessed with Pittsburgh Sleep Quality Index score and excessive daytime sleepiness assessed by Epworth sleepiness scale (ESS) $(\boldsymbol{p}<\mathbf{0 . 0 5})$. Which means that the worst is quality of sleep (Higher PSQI scores) the more increase in the functional disability among our patients group.

The same for hypersomnia; the more increase in ESS score the more increase in EDSS score; which is clinically meaningful.. In the contrary; EDSS had inverse correlation with total sleep time and sleep efficiency index on PSG $(\boldsymbol{p}<0.05)$ (Table 6) and Figures (1, 2 and3).

\section{CONCLUSION}

Sleep disorders are prevalent among MS patients. PPMS and SPMS patients show more prevalence of sleep disorder than RRMS with poorer sleep parameters on PSG. The poorer the sleep quality of MS patients the higher is the functional disability. The lower the sleep efficiency on PSG the more is the functional disability. 


\section{REFERENCES}

1. Compston A, Coles A (2002): Multiple sclerosis. Lancet, 359:1221-31.

2. Trapp BD, Peterson J, Ransohoff RM, Rudick R, Mork S, Bo L (1998): Axonal transection in the lesions of multiple sclerosis. N Engl J Med., 338:278-85.

3. Farez MF, Balbuena Aguirre ME, Varela F, Kohler AA, Correale J (2014): Autoimmune disease prevalence in a multiple sclerosis cohort in Argentina..doi: $10.1155 / 2014 / 828162$.

4.Chitnis T, Glanz B, Jaffin S, Healy B (2009): Demographics of pediatric onset multiple sclerosis in an MS center population from the Northeastern United States. Mult Scler., 15(5):627-31.

5. World Health Organization (2008): Atlas multiple sclerosis resources in the world 2008. Geneva: WHO Press.

6. Evans C, Beland SG, Kulaga S, Wolfson C, Kingwell E, Marriott J et al. (2013): Incidence and prevalence of multiple sclerosis in the Americas: a systematic review. Neuroepidemiology,40(3):195-210.

7. Young T, Palta M, Dempsey J, Skatrud J, Weber S, Badr S(1993): The occurrence of sleep-disordered breathing among middle-aged adults. N Engl J Med., 328(17):1230-5.

8. Berger K, Luedemann J, Trenkwalder C, John U, Kessler C(2004): Sex and the risk of restless legs syndrome in the general population. Arch Intern Med.,164(2):196-202.

9. Ohayon M(2002): Epidemiology of insomnia: what we know and what we still need to learn. Sleep Med Rev.,6:97-111.

10. Manconi M, Ferini-Strambi L, Filippi M, Bonanni E, Iudice A, Murri L et al. (2008): Multicenter case-control study on restless legs syndrome in multiple sclerosis: the REMS Study. Sleep, 31:944-52.

11. Nishino S, Kanbayashi T (2005): Symptomatic narcolepsy, cataplexy and hypersomnia, and their implications in the hypothalamic hypocretin/orexin system. Sleep Med Rev., 9(4):269-310.

12. Veauthier C, Radbruch H, Gaede G, Pfueller CF, Dorr J, Bellmann- Strobl J et al. (2011): Fatigue in multiple sclerosis is closely related to sleep disorders: a polysomnographic cross-sectional study. Mult Scler., 17(5):613-22.

13. Polman CH, Reingold SC, Banwell B , Clanet M, Cohen JA, Filippi M (2011): Diagnostic Criteria for Multiple Sclerosis:2010 Revisions to the McDonald Criteria .Ann Neurol.,69:292-302.

14. Kurtzke JF (2008): Historical and Clinical Perspectives of the Expanded Disability Status Scale. Neuroepidemiology,31:1-9.

15. Broderick JE, Junghaenel DU, Schneider S,Pilosi JJ, Stone AA (2013): Pittsburgh and Epworth Sleep Scale items: accuracy of ratings across different reporting peroids. Behav Sleep Med.,11:173-188.

16. The International Restless Legs Syndrome Study Group (2003): Validation of the International Restless Legs Syndrome Study Group Rating Scale for Restless Legs Syndrome.Sleep Med.,4(2):121-132.

17. Rechtschaffen A, Kales A(1968): A Manual of Standardised Terminology, Techniques and Scoring System for Sleep Stages of Human Subjects. https://www.ncbi.nlm.nih.gov/nlmcatalog/?term=Rechtschaff en $\% 20 \mathrm{~A}, \% 20 \mathrm{Kales} \% 20 \mathrm{~A} \%$
18. World Health Organization (1992): The ICD-10 classification of mental and behavioral disorders. Clinical descriptions and diagnostic guidelines. Geneva: World Health Organization.

19. American Academy of Sleep Medicine (2014): International Classification of Sleep Disorders, $3^{\text {rd }}$ ed: Diagnostic and Coding https://www.ncbi.nlm.nih.gov/pubmed/25367475

20. Veauthier C (2015): Sleep Disorders in Multiple Sclerosis. Review Curr Neurol Neurosci Rep.,15: 21.

21. Brass SD, Li CS, Auerbach S (2014): The under diagnosis of sleep disorders in patients with multiple sclerosis. J Clin Sleep Med., 10(9):1025-31.

22. Leonavicius R, Adomaitiene V (2014): Features of sleep disturbances in multiple sclerosis patients. Psychiatr Danub., 26 (3):249-55.

23. Tachibana N, Howard RS, Hirsch NP (1994): Sleep problems in multiple sclerosis. Eur Neurol., 34:320-3.

24. Borel JC, Borel AL, Monneret D, Tamisier R, Levy P, Pepin JL (2012): Obesity hypoventilation syndrome: from sleep-disordered breathing to systemic comorbidities and the need to offer combined treatment strategies. Respirology, 17(4):601-10.

25. Khan MT, Franco RA (2014): Complex sleep apnea syndrome. Sleep Disorders ,79:84-87.

26. Veauthier C, Paul F (2014): Sleep disorders in multiple sclerosis and their relationship to fatigue. Sleep Med.,15(1):514.

27. Kaminska M, Kimoff RJ, Benedetti A, Robinson A, BarOr A,Lapierre Y et al. (2012):Obstructive sleep apnea is associated with fatigue in multiple sclerosis. Mult Scler., 18(8):1159-69.

28. Iber C, Ancoli-Israel S, Chesson Jr AL (2007): The AASM Manual for the scoring of sleep and associated events: rules, terminology and technical specifications. Westchester: American Academy of Sleep Medicine. https://static1.squarespace.com/static/.../t/.../Sleep+Stage+Sco ring+c3+version.pdf

29. American Academy of Sleep Medicine Task Force (1999): Sleep-related breathing disorders in adults: recommendations for syndrome definition and measurement techniques in clinical research. Sleep, 22:667-89.

30. Kallweit U, Baumann CR, Harzheim M, Hidalgo $H$, Pohlau D, Bassetti CL et al.(2013): Fatigue and sleepdisordered breathing in multiple sclerosis: a clinically relevant association? Mult Scler Int., 2013:286581.

31. Braley TJ, Chervin RD, Segal BM (2012): Fatigue, tiredness, lack of energy, and sleepiness in multiple sclerosis patients referred for clinical polysomnography. Mult Scler Int.,67:39-36.

32. Chen JH, Liu XQ, Sun HY, Huang Y (2014): Sleep disorders in multiple sclerosis in China: clinical, polysomnography study, and review of the literature. J Clin Neurophysiol.,31(4):375-81.

33. Braley TJ, Segal BM, Chervin RD (2012): Sleep-disordered breathing in multiple sclerosis. Neurology., 79(9):929-36.

34. Dauvilliers Y, Winkelmann J (2013): Restless legs syndrome: update on pathogenesis. Curr Opin Pulm Med., 19(6):594-600.

35. Winkelman JW (2007): Periodic limb movements in sleep; endophenotype for restless legs syndrome? N Engl J Med., 357(7):703-5. 